\title{
A heterogeneous short-range communication platform for Internet of Vehicles
}

\author{
Naser Zaeri \\ Faculty of Computer Studies, Arab Open University, Kuwait
}

\begin{tabular}{l}
\hline Article Info \\
\hline Article history: \\
Received Apr 29, 2020 \\
Revised Sep 13, 2020 \\
Accepted Oct 11, 2020 \\
\hline
\end{tabular}

\section{Keywords:}

Bluetooth

Internet of vehicles

Sensors

Short-range communication

Wi-Fi connectivity performance

\begin{abstract}
The automotive industry is rapidly accelerating toward the development of innovative industry applications that feature management capabilities for data and applications alike in cars. In this regard, more internet of vehicles solutions are emerging through advancements of various wireless medium access-control technologies and the internet of things. In the present work, we develop a short-range communication-based vehicular system to support vehicle communication and remote car control. We present a combined hardware and software testbed that is capable of controlling a vehicle's startup, operation and several related functionalities covering various vehicle metric data. The testbed is built from two microcontrollers, Arduino and Raspberry Pi 3, each of which individually controls certain functions to improve the overall vehicle control. The implementation of the heterogeneous communication module is based on the IEEE 802.11 and IEEE 802.15 medium access control technologies. Further, a control module on a smartphone was designed and implemented for efficient management. Moreover, we study the system connectivity performance by measuring various important parameters including the coverage distance, signal strength, download speed and latency. This study covers the use of this technology setup in different geographical areas over various time spans.
\end{abstract}

This is an open access article under the CC BY-SA license.

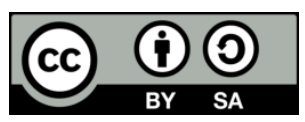

Corresponding Author:

Naser Zaeri

Faculty of Computer Studies

Arab Open University, P.O. Box 830 Ardiya, 92400, Kuwait

Email: n.zaeri@aou.edu.kw

\section{INTRODUCTION}

Recently, the internet of vehicles (IoV) has attracted researchers and engineers in the vehicle industry and manufacturing. It is aimed to be an important component of the forthcoming intelligent transportation system architecture that facilitates several functions expected to take place by the driver in such a manner that it extends the driver's ability to use on-board devices (e.g., radar or sensors) and, thus, improve road traffic safety and efficiency [1]. Vehicle telemetry and metric data can include those concerning or from the global positioning system (GPS), vehicle operations, engine performance and the engine control system. The engine control system in particular can exhibit the ability to diagnose, record, monitor, control and or optimise engine performance [2].

Actually, the IoV encompasses two main technology directions [3], vehicles' networking and vehicles' intelligence. Vehicle networking is a term used to define a connected vehicle interchanging electronic data and providing such information services as location-based information services, remote diagnostics, on-demand navigation and audiovisual entertainment content. On the other hand, vehicles' intelligence seeks to integrate the driver's technological skills and the vehicle's capabilities together as a 
single unit. This is accomplished by proposing advanced concepts such as deep learning, and cognitive computing. In other words, IoV is an open and integrated network system with high manageability, controllability and operationalisation that can involve multiple users, multiple vehicles, multiple components and multiple networks [4].

In this work, we present a combined hardware and software testbed that is capable of controlling a vehicle's start-up, windows control and door security lock capacities and several related functionalities covering various vehicle metric data. The testbed is built from two microcontrollers, Arduino [5] and Raspberry Pi 3 [6], each of which controls certain functions to enhance the overall performance of the vehicle' system. Meanwhile, both the controllers and the associated functions are controlled and driven by a wireless communication system using two different technologies: Wi-Fi [7] and Bluetooth [8]. Further, the proposed testbed is equipped with a computer-controlled centre console, allowing it to warn of hazards in road traffic and enable the driver to better manage several inter-vehicle functions ranging from music to navigation and climate systems using a smartphone. Moreover, we herein present a detailed study of the WiFi transmission and connectivity performance of the proposed system. This investigation covers the system's deployment in different geographical areas over various time spans, where we measure various important factors and parameters that are vital to accurately assessing the performance of such a wireless communication system. These parameters include the coverage distance, signal strength, download speed, and latency.

The rest of this paper is organised as follows. Related work is briefly discussed in section 2 . Section 3 covers the wireless medium access control technologies. Section 4 discusses the evolution of Wi-Fi and its effects on the IoV. The proposed system architecture is provided in section 5. The experimental results are presented and discussed in section 6. Finally, the paper is brought to a conclusion in section 7 .

\section{RELATED WORK}

Shaokun et al. [9] studied the ARM9-structured processor and embedded Linux system as means to drive improvements in the versatility and real-time capacity of a wireless remote-control car. The system collected data using sensors and transmitted them to a PC console and the authors demonstrated that the system can accomplish multiple tasks under safeguards in a timely fashion. Liu et al. [10] proposed adopting vehicle communication methods to improve safety by way of wireless data exchange. The communication structure is based on a message being sent that can be derived using non-vehicle-based technologies such as GPS to identify the location and speed of a vehicle or by employing vehicle-based sensor data wherein the location and speed data are derived from the vehicle's computer and combined with other data such as latitude, longitude or angle to produce a richer, more detailed situational awareness of the positioning of other vehicles. To enhance the performance of vehicle communication, a new variation of wireless access in the vehicular environment has been developed [11] that is capable of supporting a range of applications and services belonging to intelligent transportation systems. Zheng et al. [12] used long-range Wi-Fi that can provide improved radio coverage of up to more than $1 \mathrm{~km}$ as compared with other related options. Fangchun et al. [3] discussed the mobility constraints related to vehicular networks, where the number of connected vehicles is a major issue in this regard. Several characteristics of large cities, such as traffic jams, tall buildings, poor behaviour by drivers and complex road networks, further complicate this matter. Also, Yuan et al. [13] evaluated the performance of the IEEE 802.11 medium access control (MAC) protocol applied to vehicle safety communications in a typical highway environment.

Lwin et al. [14] discussed signal propagation from an IEEE 802.11n access point being nonuniform in the circumferential and height directions due to the multiple input-multiple output (MIMO) system of antennas. As per their discussion, the data transmission speed between the access point and a host could be significantly affected as a result of this and, to solve this problem, these authors proposed a minimax approach for optimizing the setup condition in terms of the angles and the height in an indoor environment using throughput measurements. Elsewhere, Raza et al. [15] proposed a new open-hardware platform for Bluetooth low energy to be incorporated into internet of things (IoT) systems, while Xu et al. [16] described an improved Wi-Fi method to replace GPS behaviour for the indoor environment, using a raw-data smoothing technique with an ensemble classification neural network method to deal with noisy Wi-Fi signal strength.

\section{WIRELESS MEDIUM ACCESS CONTROL TECHNOLOGIES}

Currently, there are different approaches to developing wireless MAC (the layer that controls the hardware responsible for interaction with the media) for inter-vehicle communication that differ in terms of the adopted radio interface. In this section, we briefly outline the main MAC technologies and their potential. 


\subsection{Bluetooth}

Bluetooth is a short-range wireless technology based on the IEEE 802.15.1 standard operating in the industrial, scientific and medical (ISM) frequency band $(2.4 \mathrm{GHz})$. It allows for the communication between portable devices at a data rate of up to $3 \mathrm{Mbps}$ and is highly commercialised for consumer electronics using a spread spectrum, frequency-hopping full-duplex signal at a nominal rate of 1,600 hops/s [17]. Bluetooth uses less power and costs less to implement relative to Wi-Fi. Its lower power makes it far less prone to suffering from or causing interference with other wireless devices operating in the same 2.4-GHz radio band. These advantages come at the expense of range and transmission speeds, however, which are typically lower than those of Wi-Fi. Moreover, due to its poor scalability, a Bluetooth network can only support eight active devices at maximum. Bluetooth devices are common in current automobiles, such as Bluetooth headsets and rearview mirrors [8].

\subsection{ZigBee}

One option for enabling IoV connectivity is through the use of ZigBee technology, which is based on the IEEE 802.15.4 standard and which operates on the ISM radio spectrum [18]. ZigBee is low-cost and can provide an acceptable data rate $(250 \mathrm{Kbps}$ in the $2.4 \mathrm{GHz}$ frequency band).

\subsection{Radio-frequency identification (RFID)}

The feasibility of using RFID technology for building intra-vehicle sensor networks has been studied by the ZigBee alliance [19]. In this technology, each sensor is equipped with an RFID tag and a reader connected to the vehicle's electrical control unit periodically retrieves the sensed data by sending an energizing pulse to each tag [20].

\subsection{Ultra-wideband}

Ultra-wideband (UWB) refers to radio technology that operates in the 3.1 to $10.6 \mathrm{GHz}$ frequency band and that can support short-range communications at a data rate of up to $480 \mathrm{Mbps}$ and at a low energy level [21]. UWB systems offer a number of advantages, such as resistance to severe wireless channel fading as well as a high time-domain resolution suitable for localisation and tracking applications [22]. However, proposing appropriate channel models able to capture the propagation characteristics of in-vehicle environments, is a major challenge as the channel statistics are quite different from location to location. Defining the most suitable transmission techniques at the physical (PHY) layer is a critical issue for UWBbased intra-vehicle sensor networks [23].

\subsection{Cellular technology using 3G/4G/LTE}

The cellular technology extensions have the potential to support high granularity for data transmission and flexible assigning of radio resources due to the CDMA/OFDMA component, yet suffer from the complexity of designing a coordination function in an ad-hoc mode [24]. It should be noted that this direction of research is accelerating rapidly in parallel with the Wi-Fi direction, especially with the emerging adoption of $5 \mathrm{G}$ networks.

\subsection{Wi-Fi}

Wi-Fi technology, based on the IEEE wireless communication standard 802.11, has continually improved, with each generation bringing about faster speeds, lower latency and enhanced user experiences in a multitude of environments and with a variety of device types [25]. Basically, Wi-Fi enables networking between computers and digital devices without the need for physical wires; instead, using high frequencies, radio technologies can send data seemingly through the air over short distances. Devices adhering to the 802.11 standard run on either $2.4 \mathrm{GHz}$ or $5 \mathrm{GHz}$, depending on their type. Several target groups have been working toward different variations of the 802.11 family (e.g., $802.11 \mathrm{a} / \mathrm{b} / \mathrm{g} / \mathrm{n} / \mathrm{ac} / \mathrm{ad} / \mathrm{ax}$ ), all of which present different characteristics and challenges that make them suitable for deployment in different environments [26]. Overall, the 802.11 standard supports short radio coverage with relatively higher data rates. The subsequent section will focus on Wi-Fi technology in more detail given that it has been adopted as the main wireless communication medium in our system.

\subsection{WiMAX}

This wireless technology is affiliated with IEEE $802.16 \mathrm{a} / \mathrm{e} / \mathrm{m}$ standards [27]. IEEE 802.16 standardbased WiMAX systems are able to cover a large geographical area, up to $50 \mathrm{~km}$ and can deliver significant bandwidth to end-users-in theory, up to $72 \mathrm{Mbps}$. While the IEEE 802.16 standard only supports fixed broadband wireless communications, the IEEE 802.16e/mobile WiMAX standard supports speeds of up to $160 \mathrm{~km} / \mathrm{h}$ and different classes of quality of service, even for non-line-of-sight transmissions. The key 
advantage of WiMAX relative to a wireless local area network (WLAN) is that the channel access method in the former employs a scheduling algorithm that the subscriber station needs to follow only once for initial entry into the network.

\section{IoV AND THE EVOLUTION OF WI-FI}

In 1999, Wi-Fi technology emerged as a promising technology to support the WLAN initiative, which can enable the implementation of various kinds of applications that touch various aspects of our lives. Several visionary companies came together to form a global nonprofit association with the goal of driving the development and adoption of new wireless networking technology. In 2000, the group adopted the term 'Wi-Fi' as the proper name for its technical work and announced its official name: the Wi-Fi alliance [7]. The term 'Wi-Fi' is used to describe a certified wireless networking product conforming to an industry standard designated by the IEEE. Since 1999, various versions of Wi-Fi technologies have been proposed and developed, with the early versions including IEEE802.11a, 802.11b and 802.11g. However, by 2009 and the first launch of the 802.11n protocol, alongside consequent advancements in the technology, applications of the IoV began to emerge as feasible. As such, we offer a brief description of IEEE $802.11 \mathrm{n}$ and its successor versions below.

\subsection{IEEE 802.11n}

The IEEE $802.11 \mathrm{n}$ standard operates in the 2.4-GHz and 5-GHz bands and provides good coverage across the home and office for everyday functions that do not require extensive amounts of bandwidth such as browsing the Internet and managing smart home products [26, 28]. At this point, 802.11n devices have become key enablers of smart living applications. Many of the IoT devices available now, from wearables to televisions, have adopted this standard. Also, it offers a longer reach relative to those of preceding wireless technologies and can support many client devices without sacrificing signal strength. Also, the power-saving feature ensures longer sleep periods and reduces the need for devices to communicate with infrastructure too frequently.

\subsection{IEEE 802.11ad}

The 802.11ad specification for the wireless transmission of data (originally for video-streams) in the $60-\mathrm{GHz}$ band provides speeds in the multigigabit range [29]. In the range around $60 \mathrm{GHz}$, an unlicensed frequency band between 57 and $64 \mathrm{GHz}$ is available that permits higher channel bandwidths for greater throughput and can provide global availability and avoid the overcrowded 2.4-GHz and 5-GHz bands. In fact, it can support data rates of up to $8 \mathrm{Gbit} / \mathrm{s}$ and operate at different modes-for example, during energy-saving mode for battery-operated devices and high-performance mode for very high throughput.

One of its anticipated applications is its use in small-cell backhaul [30]. Backhaul is the transmission link between the small cell and the mobile network controller. Small-cell controllers can be deployed in the streets (e.g., lampposts, streetlights), where their deployment cost is small as compared with that of optical fibre. Another advantage is their small wavelengths (approximately $5 \mathrm{~mm}$ ). These make it possible to use compact and competitive antennas or antenna arrays for beamforming, which can be used to both focus power to the receiver and help in optimizing power at the receiver. Further, beamforming can provide the necessary antenna gain to compensate for the high free-space pass loss. This efficiently helps in overcoming interference during transmission in real-time.

However, this range also has its disadvantages. For example, the field attenuation in this band is very high, topping $68 \mathrm{~dB}$ after $1 \mathrm{~m}$ and even $90 \mathrm{~dB}$ after $10 \mathrm{~m}$ of travelling [31]. Nonetheless, the high degree of attenuation can also be seen as an advantage because the transmission typically takes place within a limited range (within $4 \mathrm{~m}$ ), which means that interference from adjacent transmissions is more unlikely; as such, the transmission becomes more secure.

\subsection{IEEE 802.11ac}

Through the use of MIMO antenna technology, this standard can reduce error and boost the speed. The IEEE 802.11ac standard supports data rates of up to $3.46 \mathrm{Gbps}$ and supports $40 \mathrm{MHz}, 80 \mathrm{MHz}$ and $160 \mathrm{MHz}$ channel bandwidths as compared with just the $20 \mathrm{MHz}$ and $40 \mathrm{MHz}$ bandwidths supported by 802.11n. Further, 802.11ac supports up to eight spatial streams in contrast with the maximum of four supported with $802.11 \mathrm{n}$. The PHY data subcarriers are modulated using binary phase-shift keying, quadrature phase-shift keying, 16-quadrature amplitude modulation (QAM), 64-QAM and 256-QAM [32]. 


\subsection{IEEE 802.11ax}

This standard delivers improvements and new features that enable Wi-Fi devices to operate efficiently in the densest and most dynamic connectivity settings. IEEE 802.11ax allows enterprises and service providers to support new and emerging applications on the same WLAN infrastructure while delivering a higher grade of service to older applications [33]. This scenario sets the stage for new business models and increased Wi-Fi adoption and also powers more predictable performance for advanced applications such as $4 \mathrm{~K}$ video, ultra-high-definition, and IoT. Flexible wake-up time scheduling lets client devices sleep much longer than with 802.11ac and wake up to less contention, extending the battery life of smartphones, IoT and other devices. The PHY data subcarriers in IEEE 802.11ax are modulated using 1024QAM. IEEE 802.11ax achieves its benefits by highlighting the following dimensions:

- Orthogonal frequency division multiple access (OFDMA), which effectively shares channels to increase the network efficiency and lower latency for both uplink and downlink traffic in high-demand environments [34]

- Multi-user MIMO, which allows more downlink data to be transferred at one time, enabling access points to concurrently handle more devices

- $160-\mathrm{MHz}$ channel use capability, which increases the bandwidth needed to deliver greater performance with low latency

- Transmit beamforming, which enables higher data rates at a given range to increase network capacity

- Robust high-efficiency signalling for better operation at a significantly lower received-signal-strengthindication (RSSI)

Of these, OFDMA is especially beneficial for conducting multiple short transmissions in wide channels since many data transmissions follow the same PHY header and channel access timeout. Moreover, OFDMA allows better coping with fading and, improves the spectral power density. The efficiency of OFDMA depends upon how the access point schedules channel resources among the different stations and on how it configures other transmission parameters, such as the transmission power. Table 1 summarises some important characteristics of Wi-Fi versions including the earlier versions-namely, $802.11 \mathrm{a} / \mathrm{b} / \mathrm{g}$. CCK, which stands for complementary code keying, was a short-lived deviation that existed prior to the huge advancements reached by OFDM in later versions.

Table 1. Wi-Fi versions

\begin{tabular}{cccccc}
\hline Standard & Year & Band/GHz & Modulation method & Highest modulation order & Highest code rate \\
\hline $802.11 \mathrm{a}$ & 1999 & 5 & OFDM & $64-\mathrm{A} M$ & $3 / 4$ \\
$802.11 \mathrm{~b}$ & 1999 & 2.4 & CCK & QPSK & $1 / 2$ \\
$802.11 \mathrm{~g}$ & 2003 & 2.4 & OFDM & $64-\mathrm{AM}$ & $3 / 4$ \\
$802.11 \mathrm{n}$ & 2009 & $2.4 / 5$ & OFDM & 64-QAM & $5 / 6$ \\
$802.11 \mathrm{ad}$ & 2012 & 60 & OFDM & 64-QAM & $13 / 16$ \\
$802.11 \mathrm{ac}$ & 2014 & 5 & OFDM & 256-QAM & $5 / 6$ \\
$802.11 \mathrm{ax}$ & 2019 & $2.4 / 5$ & OFDM & 1024-QAM & $5 / 6$ \\
\hline
\end{tabular}

\section{SYSTEM ARCHITECTURE}

Different from generic wireless sensor networks, intra-vehicle wireless sensor networks show unique characteristics that provide the space for optimisation. For example, the sensors in intra-vehicle networks are stationary so that the network topology does not change over time. Also, these sensors are typically connected through one hop, which yields a simple star-topology. Further, with this arrangement, there is no energy constraint for sensors having wired connections to the vehicle power system.

A typical IoV architecture consists of the following three layers:

- The perception layer, which contains all sensors within the vehicle that gather data and detect specific events of interest; it also boasts RFID, satellite-positioning perception, road-environment perception, vehicle-position perception and other-object-placement perception capabilities.

- The network layer, which is the communication layer that ensures connectivity to communications networks such as 3G/4G/5G, WiMax, WLAN, Wi-Fi and Bluetooth.

- The application layer, which is responsible for storage, analysis, processing and decision-making concerning different risk situations such as traffic congestion and bad weather; it also encompasses smart applications, traffic safety, efficiency and multimedia-based infotainment components.

We herein propose a system that is composed of two microprocessors (Arduino and Raspberry Pi 3) monitored by an Android-based platform. In this arrangement, both microprocessors and the associated functions are governed by wireless communication systems using Wi-Fi and Bluetooth technologies. In addition, the system is connected to a touchscreen monitor and a microphone to minimise driving distraction 
and ease car control via the use of voice. Figure 1 shows the proposed vehicle communication system architecture. As demonstrated in Figure 1, each one of the two microcontrollers controls certain functionalities. The Raspberry Pi 3 microprocessor, shown in Figure 2, uses Samsung's (Seoul, South Korea) S5 CPU 1.2-GHz 64-bit plus $1 \mathrm{~GB}$ of RAM and enables Bluetooth and Wi-Fi connections. This processor requires a power adapter to feed it with a steady 2.5-A current for proper performance. Meanwhile, the Raspberry Pi control system consists of the SDK library, which provides support for all Android auto features. Also, it provides support for the asynchronous mechanisms inherent in communication, multimedia, user input and graphical interface actions. The system uses OpenSSL for encrypting communication. The Raspberry Pi microcontroller controls and supports components of the vehicle's built-in infotainment system such as audio, phone calls and navigation.

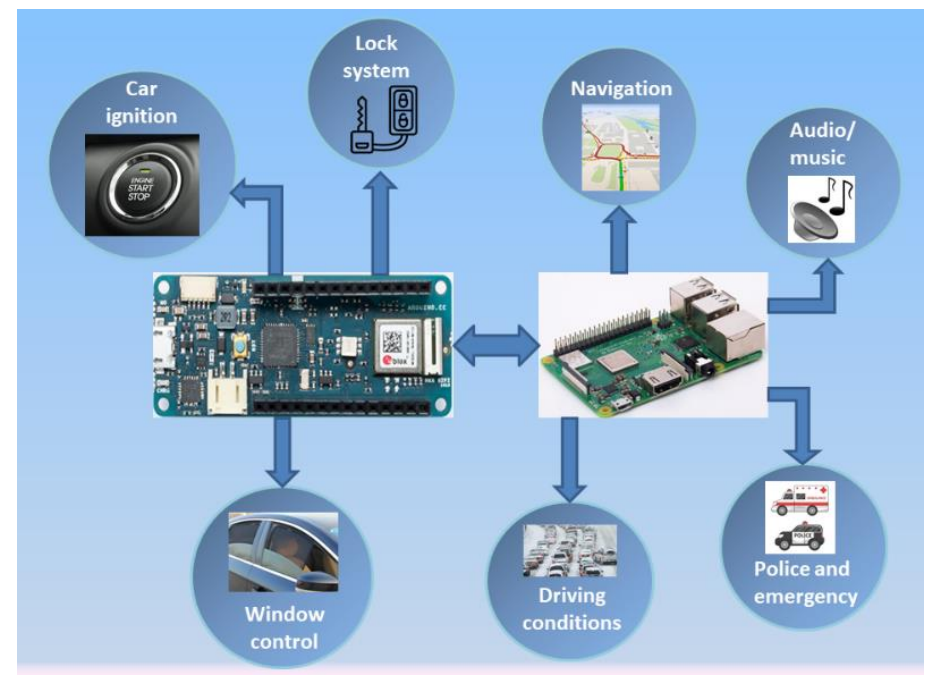

Figure 1. The proposed vehicle system communication architecture

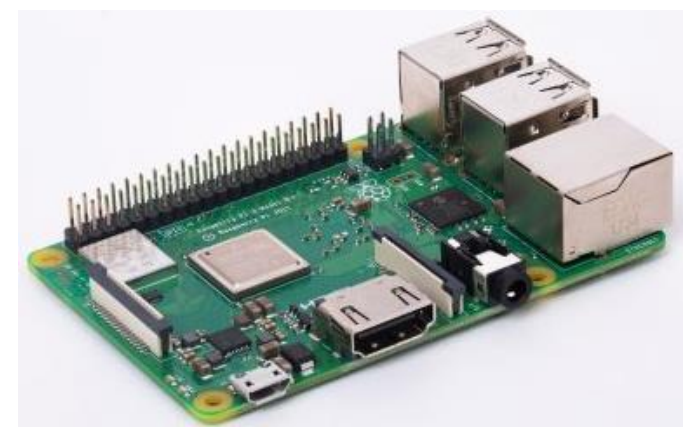

Figure 2. Raspberry Pi 3 microprocessor

The other microprocessor is the Arduino one. Arduino, shown in Figure 3, is an open-source computer hardware and software platform that supports the design and creation of mini-controllers [5]. Further, it enables Bluetooth and Wi-Fi connections. The Arduino microprocessor is connected to a relay as shown in Figure 4 that controls the electrical circuit board. This board is connected to a $12-\mathrm{V}$ battery. The ignition wire and vehicle windows' electricity wires are connected to this board as well.

The relay used in this design was a four-channel relay module. In this arrangement, the switching mechanism is deployed out with the help of an electromagnet. The relay cards are connected to act as an Hbridge drive. One end of the relay card is connected to the microcontroller and the other end is connected to the terminals of the motor of the window in the car arm. The forward, stop and reverse motions of the window are controlled using the relay card, i.e., the supply voltage to the motor can be changed in either of the directions, causing clockwise and anti-clockwise motions of the motor. Further, the system is connected to an $800 \times 480$ display as shown in Figure 5 via an adapter board, which handles power and signal 
conversion. The touchscreen display supports an on-screen keyboard for full functionality without a physical keyboard or mouse. Finally, a microphone is connected to the system to enable handling voice commands.

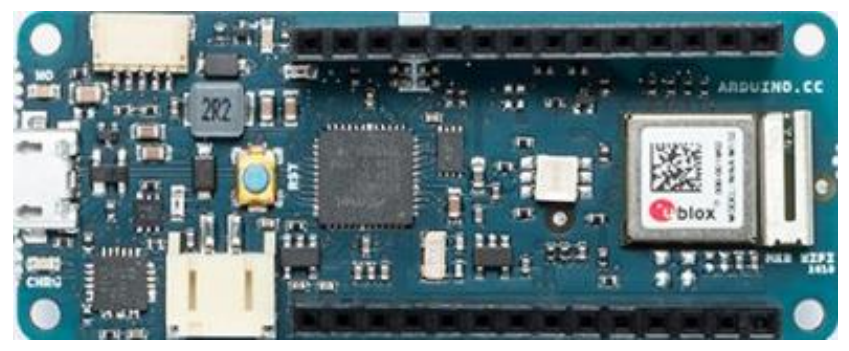

Figure 3. Arduino microprocessor

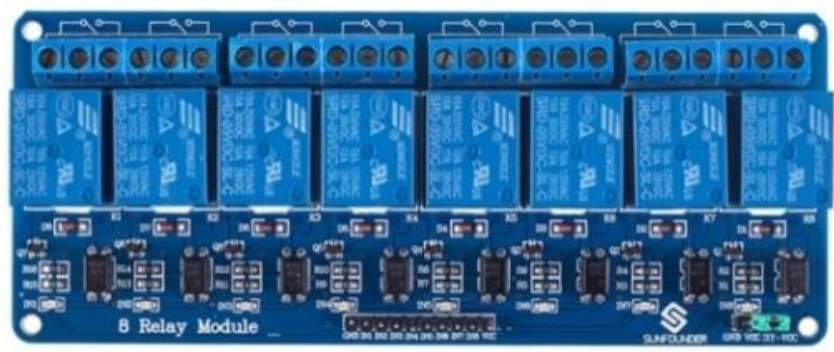

Figure 4. The electrical relay used in the Arduino board connection

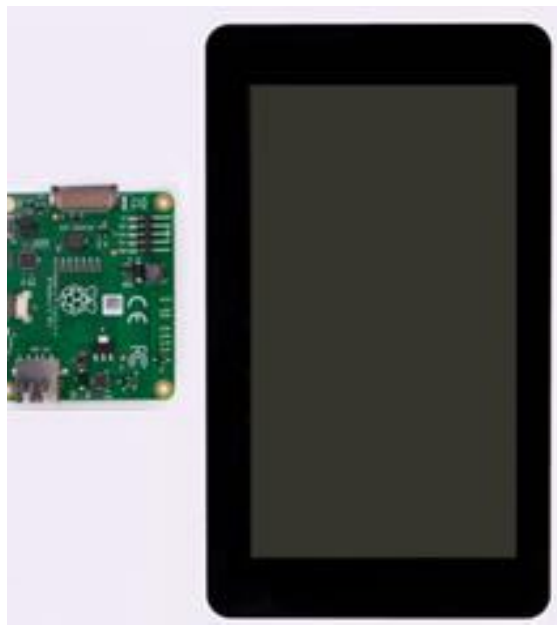

Figure 5. $800 \times 480$ display used in system control

\section{EXPERIMENTAL RESULTS AND DISCUSSION}

We began this investigation by installing the corresponding operating system on the two microprocessors. Regarding the Raspberry Pi 3, the Raspbian operating system comes preinstalled with Python, Scratch, Sonic Pi and Java. As for the Arduino microprocessor, it is configured using the C programming language. Several functionalities were coded and implemented. First, the system was configured to handle all notifications, calls and emergency calls. If a call is received, the system will automatically reply with a preset message. Also, it can handle many popular applications such as Google Hangouts, Facebook messenger and WhatsApp. Second, the system can be synchronised with the mobile phone to migrate the user's contacts, calendars, music library and saved locations. Third, a navigation system has been launched on the system, where the driver can use Google Maps in an easy manner through voice control. The voice control enables the driver to enter new destinations, set favourite addresses, stop route 
guidance and set up auto-navigation. In addition, checking and reading emails and SMS text messages is accomplished in a similar manner. The abovementioned functionalities are controlled remotely through an interface using a touchpad/mobile phone via the Bluetooth 802.15 technology. Furthermore, the system is configured to control the following functions remotely using the Wi-Fi 802.11 technology:

- Starting and turning off the engine remotely without a key

- Unlocking /locking the doors

- Pulling windows up and down

Moreover, the system enables the user to set a password of his/her choice to keep the system protected. A theft alarm is integrated into the system that alerts when an unusual activity is sensed or when a wrong password is attempted to unlock the system. To achieve this, the car ignition wire is connected to the on-board diagnostics (OBD) port of the car. If a correct password is entered, then the system will verify it and an acknowledgement (ACK) is sent. Hence, a wireless connection is established between the two control units and the remote console can perform the functions accordingly. The system interface for the Arduino functions is shown in Figure 6.

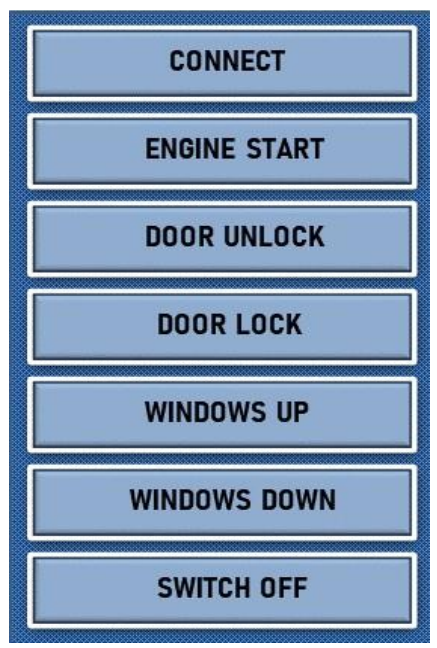

Figure 6. System interface for the Arduino functions

To test the visibility of the system and assess its performance, we conducted extensive experimental studies, focusing on the three most important parameters in any wireless communication system-that is, we studied the signal strength, download speed and latency. The performance of a wireless communication system, including the system at hand, is technically evaluated by assessing how efficient these parameters can perform in real-life practical scenarios. With respect to radio-frequency, signal strength refers to the transmitter power output as received by a reference antenna at a distance from the transmitting antenna. For very-low-power systems, such as mobile phones, signal strength is usually expressed in $\mathrm{dBm}$, which stands for decibels relative to a milliwatt. The signal strength required for optimal performance varies according to many factors such as background noise in the environment, the amount of clients on the network, what the desired data rates are and what applications will be used.

Moreover, network speed is measured by how much data the connection can download (download speed) or upload (upload speed) per second. It indicates the total maximum data-carrying capacity of the network in optimum conditions. Generally, a little over half of this capacity is consumed by management and control traffic. The remaining capacity is available for user data. This capacity is referred to as throughput and is usually expressed in bits per second (bit/s). For most applications, having fast download speeds reflect to a far extent the successful implementation of the network. This is much more readily apparent in common activities such as watching streaming television, downloading music and browsing the Internet. The throughput of a wireless LAN is linked to many other factors including the data-carrying capacity, local interference, environment (obstructions), equipment setup (including antenna orientation) and the transmission power. Furthermore, the latency refers to the amount of delay (or time) it takes to send information from one point to the next. Latency is usually measured in milliseconds (ms) and is also referred to (during speed tests) as a ping rate. One of the major reasons for poor latency is nonoptimal geography, although other important reasons that may affect the latency include the transmission media and packet size. 
In measuring the abovementioned parameters, we used various mobile applications as shown in Table 2. Further, we have considered various scenarios as part of this research. Specifically, we conducted the measurements in eight different geographical locations, including some urban and suburban ones. Moreover, we operated the system on various traffic applications, including voice over internet protocol (VoIP), video-watching, web surfing and sending emails. The duration time required for each session readings setup was between one and three hours and 20 to 60 different readings were taken in each session. The readings were repeated three times for each location over a one-month span time and the average of the readings was considered to represent the final readings.

Table 2. Experimental scenario parameters

\begin{tabular}{cc}
\hline Parameters & Description \\
\hline Applications used & Wi-Fi Speed Test, Open Signal, Wi-Fi Signal Meter, \\
& Network Signal Information \\
Wireless technologies & Wi-Fi \\
Standard & IEEE 802.11 \\
Frequency bands & $2.4 \mathrm{GHz}, 5 \mathrm{GHz}$ \\
Traffic application & VoIP, video, HTTP, email \\
Scenario & Urban, suburban \\
Number of locations (test areas) & 8 \\
Number of readings per location & $20-60$ \\
Experimental time per test & $1-3$ hours \\
\hline
\end{tabular}

Figure 7 shows an example of a signal strength test for a specific area. Figure 8 shows the average readings of the signal strength in $\mathrm{dBm}$ for all eight locations. Further, an example of a download speed test is shown in Figure 9. The average download speeds for all eight locations are given in Figure 10. Finally, the corresponding average latency is given in Figure 11.

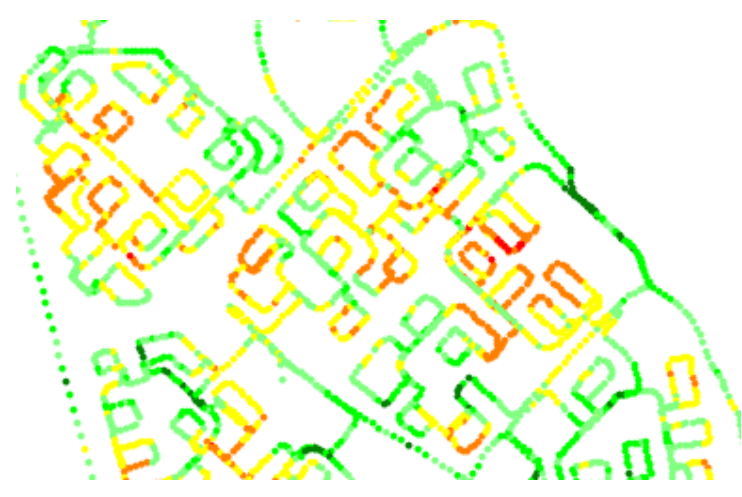

Figure 7. An example of a signal strength test for a specific area

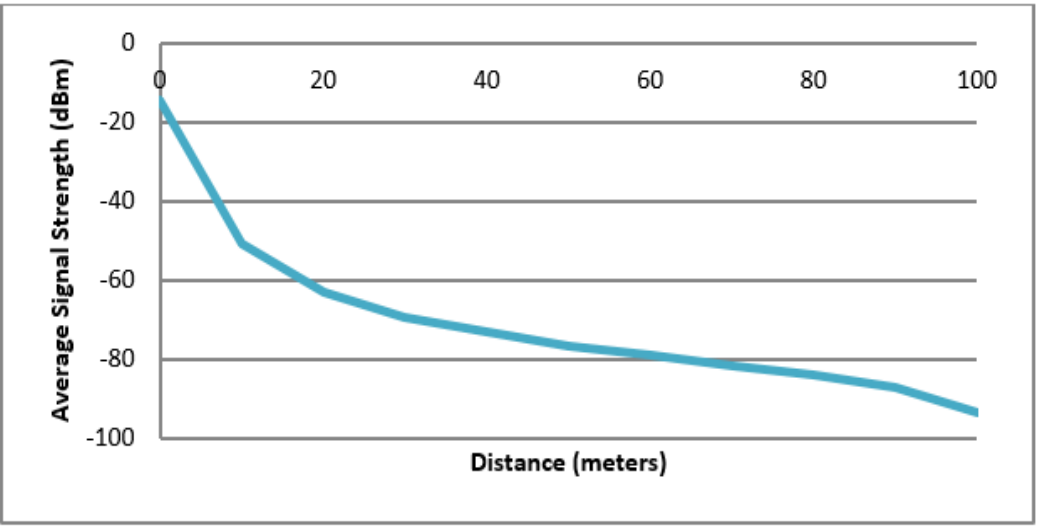

Figure 8. Average signal strength versus distance for the eight locations 


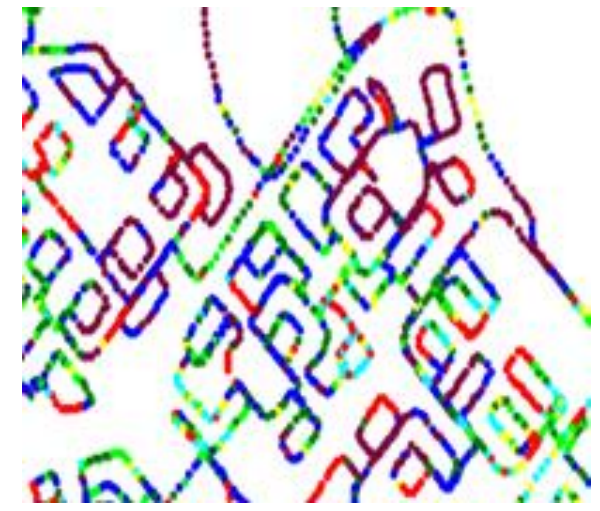

Figure 9. An example of a download speed test

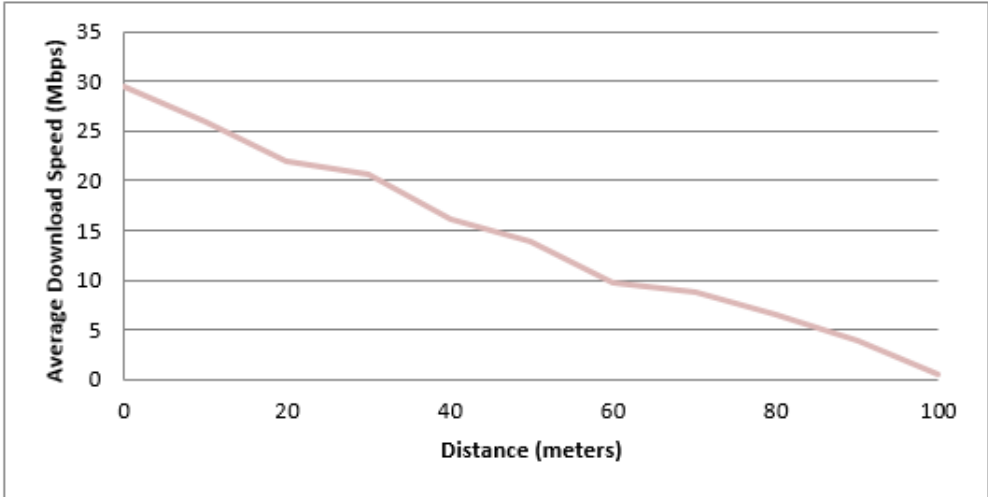

Figure 10. Average downloads speed versus distance for the eight locations

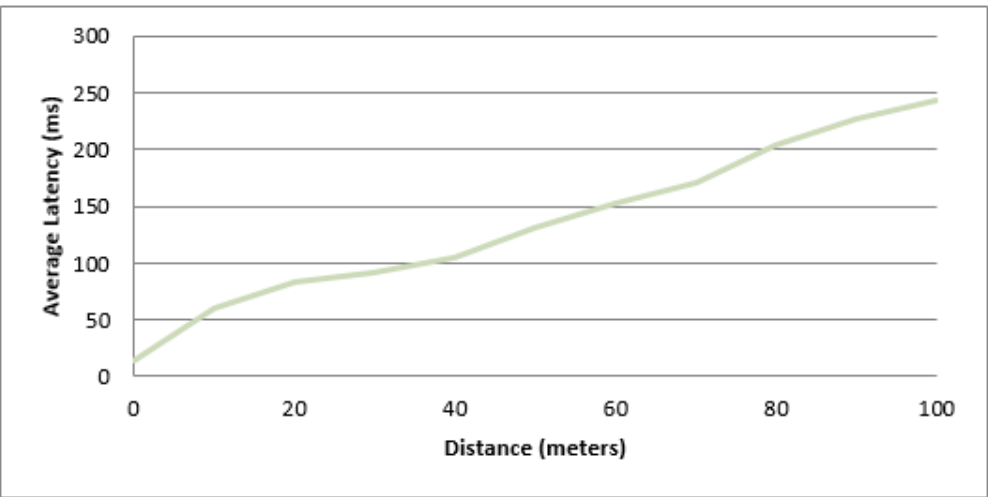

Figure 11. Average latency versus distance for the eight locations

Notably, the following remarks should be considered when viewing Figures 7 and 8 . The signal strength varies from one location to another but, in general, the system is capable of receiving signals up to almost $100 \mathrm{~m}$. Controlling the vehicle's different applications depends upon the data nature of each one of these applications. More specifically, we were able to successfully operate all the system functionalities up to $30 \mathrm{~m}$, where the strength reaches around $-65 \mathrm{dBm}$. This includes navigation, VoIP and streaming video. The connection was reliable and the timely delivery of data packets was observed. Further, the signal strength decreases to almost $-80 \mathrm{dBm}$ at a distance of $60 \mathrm{~m}$. Though this level of signal is low, some necessary applications were still able to function reliably, such as email, web browsing and the ability to call the police and emergency services. Of course, the signal strength decreased further as the distance increased from here, 
ultimately reaching levels of less than $-90 \mathrm{dBm}$ at distances of 80 meters. At this level, only two applications could successfully be controlled: the door lock system and car ignition; these control functions were maintained up to almost $100 \mathrm{~m}$.

However, some exceptions were noticed as well. For example, system performance at some locations was below the average and full successful functionalities were barely attained at a distance of $30 \mathrm{~m}$. This may be explained by the fact that specific sites can be characterised by significant traffic congestion throughout the day. In this context, the signal reflection is high and severely affects the transmission.

The above discussion regarding signal strength and system functionality performance is reinforced when we further examine Figures 9 and 10, which present the download speeds. We were able to attain an average of $6 \mathrm{Mbps}$ at distances as far as $80 \mathrm{~m}$. Actually, in two of the eight locations under study, the download speed was around 10 Mbps.

On the other hand, a noticeable variation in time latency was recognised among the various locations. For example, at a distance of $20 \mathrm{~m}$, the readings of latency varied from less than $20 \mathrm{~ms}$ to almost $200 \mathrm{~ms}$. A remarkable issue that was observed in this regard is the delay variation between the $2.4-\mathrm{GHz}$ and $5-\mathrm{GHz}$ bands. We noticed that the connection under the $5-\mathrm{GHz}$ band was more consistent than that with the $2.4 \mathrm{GHz}$ one. Nonetheless, this variation did not affect the successful operation of the various applications. In brief, these results demonstrate the successful design and implementation of the proposed system, where the combined hardware and software testbed verified an effective capability for controlling a vehicle's different functionalities.

\section{CONCLUSION AND FUTURE WORK}

In this paper, we presented a heterogeneous short-range communication platform for IoV based on IEEE 802.11 and 802.15 technologies. A combined hardware and software testbed was built to control several important vehicle functionalities including start-up, movement of windows, door security locking, hazard warnings in road traffic, navigation, climate systems and several other inter-vehicle functions. The testbed was built from two microcontrollers: Arduino and Raspberry Pi 3. Further, a control module on a smartphone was designed and implemented for efficient remote management. Moreover, we implemented extensive experimental studies on the $\mathrm{Wi}-\mathrm{Fi}$ transmission and connectivity performance of the proposed system to evaluate its efficiency in real-life practical scenarios. Our research considered several important parameters-mainly, the signal strength, download speed, and latency. All system functionalities including navigation, VoIP and video streaming successfully operated up to a 30-m distance, where the average signal strength was greater than $-65 \mathrm{dBm}$ and the average download speed was around $20 \mathrm{Mbps}$. Despite the decrease in signal strength at larger distances, the system maintained successful control of the main vehicle's functions-that is, the door lock security system and car ignition-up to $100 \mathrm{~m}$ away.

However, there remain many challenges that face IoV and slow down its full and widespread adoption. For example, IoV involves the integration of different services and standards that may attract intruders and cyber-attacks. Hence, security plays an important role in this aspect. The reliability of sensors and network coverage are other important challenges that need to be tackled by researchers to ensure proper functioning and effective signal communications in real time. These issues will be addressed in our future work, where we aim to integrate the current system with 4G LTE/5G systems.

\section{REFERENCES}

[1] Contreras-Castillo, S. Zeadally, and J. Guerrero-Ibañez, "Internet of vehicles: architecture, protocols, and security," IEEE Internet of Things Journal, vol. 5, no. 5, pp. 3701-3709, 2018, doi: 10.1109/JIOT.2017.2690902.

[2] Simon Mayer and Josh Siegel, "Conversations with connected vehicles," 5th International Conference on the Internet of Things (IOT), 2015, pp. 38-44, doi: 10.1109/IOT.2015.7356546.

[3] Yang Fangchun, Wang Shangguang, Li Jinglin, Liu Zhihan, Sun Qibo, "An Overview of Internet of Vehicles," China Communications, vol. 11, no. 10, pp. 1-15, 2014, doi: 10.1109/CC.2014.6969789.

[4] US department of Transportation, "Vehicle-to-Vehicle Communications: Readiness of V2V Technology for Application,” DOT HS 812014 Aug. 2014. [Online], Available: https://www.its.dot.gov/cv_basics/pdf/Readinessof-V2V-Technology-for-Application-812014.pdf.

[5] "What is Arduino?," [Online], Available: https://www.arduino.cc,

[6] "Raspberry Pi 3," [Online], Available: https://www.raspberrypi.org,

[7] "Wi-fi"[Online], Available: https://www.wi-fi.org/,

[8] A. Bhaskar, M. Qu and E. Chung, "Bluetooth Vehicle Trajectory by Fusing Bluetooth and Loops: Motorway Travel Time Statistics," IEEE Transactions on Intelligent Transportation Systems, vol. 16, no. 1, pp. 113-122, Feb. 2015, doi: 10.1109/TITS.2014.2328373. 
[9] Wang Shaokun, Xiao Xiao, and Zhao Hongwei, "The Wireless Remote Control Car System Based on ARM9," First International Conference on Instrumentation, Measurement, Computer, Communication and Control, 2011, doi: 10.1109/IMCCC.2011.224.

[10] Zishan Liu, Zhenyu Liu, Zhen Meng, Xinyang Yang, Lin Pu and Lin Zhang, "Implementation and performance measurement of a V2X communication system for vehicle and pedestrian safety," International Journal of Distributed Sensor Networks, vol. 12, no. 9, pp. 887-890, 2016, doi: 10.1177/1550147716671267.

[11] Gongbo Yan and Jinxu Guo, "The research on two different backoff algorithms in IEEE 802.11p mac layer," 7th IEEE International Conference on Electronics Information and Emergency Communication (ICEIEC), 2017, pp. 417-420, doi: 10.1109/ICEIEC.2017.8076595.

[12] Zhe Zheng, et al., "Discussion and Testing of 802.11ah Wireless Communication in Intelligent Substation," IEEE International Conference on Energy Internet (ICEI), 2018, pp. 208-212, doi: 10.1109/ICEI.2018.00045.

[13] Yuan Yao, et al., "Delay Analysis and Study of IEEE 802.11p based DSRC Safety Communication in a Highway Environment," Proceedings of IEEE INFOCOM, 2013, doi: 10.1109/INFCOM.2013.6566955.

[14] Kyaw Soe Lwin, et al., "A Minimax Approach for Access Point Setup Optimization in IEEE 802.11n Wireless Networks," International Journal of Networking and Computing, vol.7 no. 2, pp. 187-207, 2017, doi: 10.15803/ijnc.7.2_187.

[15] Shahid Raza, Prasant Misra, Zhitao He, and Thiemo Voigt, "Building the Internet of Things with bluetooth smart," Ad Hoc Networks, vol. 57, pp. 19-31, 2017, doi.org/10.1016/j.adhoc.2016.08.012

[16] W. Xu, H. A. Omar, W. Zhuang and X. S. Shen, "Delay Analysis of In-Vehicle Internet Access Via On-Road WiFi Access Points," IEEE Access, vol. 5, pp. 2736-2746, 2017, doi: 10.1109/ACCESS.2017.2669178.

[17] Madeline Cheah, et al., "Towards a systematic security evaluation of the automotive Bluetooth interface," Vehicular Communications, vol. 9, pp. 8-18, 2017, doi: 10.1016/j.vehcom.2017.02.008.

[18] ZigBee Alliance, “Zigbee specification,” [Online], Available: http://www.zigbee.org,.

[19] O. K. Tonguz, H.-M. Tsai, T. Talty, A. Macdonald, and C. Saraydar, "RFID technology for intra-car communications: a new paradigm," IEEE Vehicular Technology Conference, 2006, doi: 10.1109/VTCF.2006.618.

[20] O. K. Tonguz, et al., "Intra-car wireless sensor networks using RFID: Opportunities and challenges," 2007 Mobile Networking for Vehicular Environments, 2007, doi: 10.1109/MOVE.2007.4300802.

[21] I. Oppermann, M. H"am"al"ainen, and J. Iinatti, "UWB: theory and applications," Wiley, 2005

[22] C. U. Bas and S. C. Ergen, "Ultra-wideband Channel Model for Intravehicular Wireless Sensor Networks Beneath the Chassis: From Statistical Model to Simulations," IEEE Trans. on Vehicular Technology, vol. 62, no. 1, pp. 14-25, 2013, doi: 10.1109/TVT.2012.2215969.

[23] Y. Sadi and S. Ergen, "Optimal Power Control, Rate Adaptation and Scheduling for UWB-based Intra-vehicular Wireless Sensor Networks," IEEE Trans. on Vehicular Technology, vol. 62, no. 1, pp. 219-234, 2013, doi: 10.1109/TVT.2012.2217994.

[24] Ning Lu, Nan Cheng, Ning Zhang, Xuemin Shen, Jon W. Mark, "Connected Vehicles: Solutions and Challenges," IEEE Internet of Things Journal, vol. 1, no. 4, pp. 289-299, 2014, doi: 10.1109/JIOT.2014.2327587

[25] Yang Xiao and Yi Pan, "Emerging Wireless LANs, Wireless PANs, and Wireless MANs: IEEE 802.11, IEEE 802.15, 802.16 Wireless Standard Family," Wiley Telecom eBook Chapters, vol. 57, 2009

[26] Guolin Sun, Jian Zhu; Rui He, and Chuan Xiao, "Experimental study of rate-aware scheduling for 802.11n Wi-Fi network with legacy devices," IEEE Global High Tech Congress on Electronics, 2013, pp. 46-49, doi: 10.1109/GHTCE.2013.6767238.

[27] Chatelain, D., et al., "WiMAX," Boca Raton: CRC Press, 2018, doi: 10.1201/9781315219899

[28] D. Newell, P. Davies, R. Wade, P. Decaux and M. Shama, "Comparison of Theoretical and Practical Performances with 802.11n and 802.11ac Wireless Networking," 31st International Conference on Advanced Information Networking and Applications Workshops, 2017, pp. 710-715, doi: 10.1109/WAINA.2017.113.

[29] P. Kumari, J. Choi, N. González-Prelcic and R. W. Heath, "IEEE 802.11ad-Based Radar: An Approach to Joint Vehicular Communication-Radar System," IEEE Transactions on Vehicular Technology, vol. 67, no. 4, pp. 3012-3027, 2018, doi: 10.1109/TVT.2017.2774762.

[30] Lochan Verma, Mohammad Fakharzadeh, Sunghyun Choi, "WIFI ON STEROIDS: 802.11AC AND 802.11AD," IEEE Wireless Communications, vol. 20, no. 6, pp. 30-35, 2013, doi: 10.1109/MWC.2013.6704471.

[31] T. Nitsche, C. Cordeiro, A. B. Flores, E. W. Knightly, E. Perahia and J. C. Widmer, "IEEE 802.11 ad: directional 60 GHz communication for multi-Gigabit-per-second Wi-Fi [Invited Paper]," IEEE Communications Magazine, vol. 52, no. 12, pp. 132-141, Dec. 2014, doi: 10.1109/MCOM.2014.6979964.

[32] Y. Yang and X. Zhu, "A Wideband Reconfigurable Antenna With $360^{\circ}$ Beam Steering for 802.11ac WLAN Applications," IEEE Transactions on Antennas and Propagation, vol. 66, no. 2, pp. 600-608, Feb. 2018, doi: 10.1109/TAP.2017.2784438.

[33] B. Bellalta, "IEEE 802.11ax: High-efficiency WLANS," IEEE Wireless Communications, vol. 23, no. 1, pp. 38-46, Feb. 2016, doi: 10.1109/MWC.2016.7422404.

[34] D. Bankov, A. Didenko, E. Khorov and A. Lyakhov, "OFDMA Uplink Scheduling in IEEE 802.11ax Networks," 2018 IEEE International Conference on Communications (ICC), 2018, pp. 1-6, doi: 10.1109/ICC.2018.8422767. 


\section{BIOGRAPHY OF AUTHOR}

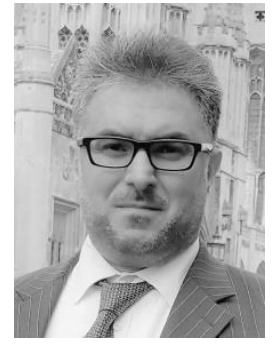

Naser Zaeri is an associate professor with the Department of Information Technology and Computing (ITC) at the Arab Open University (AOU), Kuwait. He has obtained his $\mathrm{PhD}$ in Electrical Engineering from University of Surrey, United Kingdom in 2008. He has obtained his M.Sc. and B.Sc. degrees in Electrical Engineering from Kuwait University (Honor List). He served as the Director of Research and Development at the AOU during (2012 - 2014). He was the Head of ITC Department at the AOU during (2010 - 2012). Also, he was with College of Engineering and Petroleum - Kuwait University, as a lecturer. He served as a consultant for many authorities and ministries. Dr. Zaeri has participated in and in charge of many projects in different fields of engineering and technology. He has more than 40 different publications in international journals and conferences. His areas of interest are: communications systems, digital image processing, biometrics, and pattern classification. 